\title{
Meson mixing amplitudes in asymmetric nuclear matter
}

\author{
Yoshiharu Mori晴 and Koichi Saito \\ ${ }^{a}$ Department of Physics, Tohoku University, Sendai 980-0845, Japan \\ ${ }^{b}$ Tohoku College of Pharmacy, Sendai 981-8558, Japan
}

\begin{abstract}
Using a purely hadronic model, we study the charge-symmetry-breaking $\rho$ - $\omega$, $\sigma-\delta, \sigma-\rho$ and $\delta-\omega$ mixing amplitudes in isospin asymmetric nuclear matter. The basic assumption of the model is that the mixing amplitude is generated by nucleon and anti-nucleon loops and hence driven entirely by the difference between proton and neutron Fermi momenta and the proton-neutron mass difference. We find that the behavior of the mixing amplitude is very complicated in the spacelike region and quite sensitive to the proton fraction of nuclear medium and nuclear density. In particular, in neutron rich nuclei (like $\mathrm{Pb}$ ) and/or neutron stars the mixing amplitudes become about $10 \sim 100$ times as large as those in typical mirror nuclei.

(Keywords: Charge symmetry breaking, Meson miximg, Asymmetric nuclear matter, Quantum Hadrodynamics)
\end{abstract}

PACS: 24.80.+y, 21.30.Fe, 21.65.+f

*E-mail: mori@nucl.phys.tohoku.ac.jp

${ }^{\dagger}$ ksaito@tohoku-pharm.ac.jp 
Charge independence and charge symmetry (CS) provide powerful tools in organizing and describing the multiplet structure of hadrons and nuclei. These symmetries are imperfect and, in particular, charge symmetry breaking (CSB) has been studied for a long time [1]. It is trivially broken by the electromagnetic interaction. Other well known sources are the proton $(p)$-neutron $(n)$ mass difference and the nucleon-nucleon $(\mathrm{NN})$ potential caused by meson mixing, and these sources can be related to the $u$ - $d$ quark mass difference in QCD. Thus, the small imperfection of CS provide a unique opportunity to investigate the relation between QCD and hadronic and nuclear observables.

It has long been recognized that the $\rho$ - $\omega$ mixing amplitude would give rise to the significant part of CSB in the NN force. Indeed, substantial effects have been observed in the $e^{+} e^{-} \rightarrow \pi^{+} \pi^{-}$reaction at $q^{2} \sim m_{\omega}^{2}\left(m_{\omega}=782.57 \mathrm{MeV}\right.$, the $\omega$ meson mass $)$ [1,2]. These results allow an extraction of the $\rho-\omega$ mixing amplitude, $\left\langle\rho\left|H_{\text {mix }}\right| \omega\right\rangle \simeq-4500 \mathrm{MeV}^{2}$ [2], which seems to be consistent with CSB in the NN scattering lengths [1, 2], the OkamotoNolen-Schiffer (ONS) anomaly [1.3] and the analyzing powers measured in elastic $\vec{n}-\vec{p}$ scattering 画.5.

However, about a decade ago, the importance of the momentum dependence of the $\rho-\omega$ mixing amplitude was pointed out using several different models [6.7]. It suggests that the $\rho-\omega$ mixing amplitude at spacelike momenta is different from its value at the $\omega$ pole. A QCD sum-rule calculation also gives a strong momentum dependence of the amplitude [8]. It implies that the NN potential given by the off-shell amplitude of the $\rho-\omega$ mixing is quite different from the ones used in the successful phenomenology.

On the other hand, another analysis [1] argues that the strong off-shell effect is inconsistent with the observed $q^{2}$-dependence of $\rho$ - $\gamma^{*}$ coupling (see also Ref. [9]). Moreover, Cohen and Miller [10] have emphasized that simple knowledge of the off-shell meson propagator is not sufficient to determine the CSB NN potential and that it is important to know the vertex function computed from the same theory that supplied the propagator at the same time. They have showed that the momentum dependence of the $\rho-\omega$ mixing amplitude may be absorved into a re-definition of the $\rho$ - $\mathrm{N}$ vertex. However, this problem is still controversial [1].

In this Letter, our aim is to study the properties of CSB meson mixing in isospin asymmetric nuclear matter. To understand CSB in nuclei (for example, the ONS anomaly, nuclear structure of heavy nuclei like $\mathrm{Pb}$, neutron stars etc.), it is vital to know how CSB effects depend on nuclear density $\left(\rho_{B}\right)$ and numbers of proton $(Z)$ and neutron $(N)$. Such a study has not yet been done in the spacelike region [12]. In the present calculation, we use a purely hadronic model, i.e., Quantum Hadrodynamics (QHD), to evaluate various meson mixing amplitudes in asymmetric nuclear matter.

According to Ref. [10], let us introduce a typical scale $\Lambda_{s}$ which separates the short ranged effects from the long ranged ones in meson exchange models. Then, the short ranged parts are handled by vertex functions, while the long ranged ones are explicitly treated by meson propagators. Here $\Lambda_{s}$ is taken to be of order of $1 \mathrm{GeV}$ so that $\sigma, \rho, \omega$ and $\delta$ (or $a_{0}$ ) mesons are explicitly included but heavier mesons are not. (In the present calculation, the pseudoscalar mesons are not included because we suppose the relativistic Hartree approximation (RHA).)

The Lagrangian density is thus written as

$$
\mathcal{L}=\mathcal{L}_{Q H D-I}+\mathcal{L}_{N N \delta}+\mathcal{L}_{N N \rho}+\delta \mathcal{L}_{C T},
$$


where $\mathcal{L}_{Q H D-I}$ is the usual QHD-I Lagrangian 13 which describes the $\sigma-\omega-\mathrm{N}$ system. The last term in the right hand side is a counterterm for renormalizations, which will be discussed below.

The $\delta$ meson is expressed by the Lagrangian density $\mathcal{L}_{N N \delta}$ and it interacts with the nucleon through an isovector, scalar coupling

$$
\mathcal{L}_{N N \delta}^{\text {int. }}=g_{\delta} \bar{\psi} \tau_{z} \delta \psi
$$

where $\delta$ is the neutral $\delta$-meson field and $g_{\delta}$ is the $\delta$-N coupling constant. The nucleon mass in matter $M_{i}^{*}(i=p$ or $n)$, which is a function of the $\sigma$ and $\delta$ fields, is now given by

$$
M_{\left(\begin{array}{l}
p \\
n
\end{array}\right)}^{*}=M_{\left(\begin{array}{l}
p \\
n
\end{array}\right)}-g_{\sigma} \sigma \mp g_{\delta} \delta,
$$

with $\sigma$ the $\sigma$ field, $g_{\sigma}$ its coupling constant and $M_{i}$ the free proton $\left(M_{p}=938.27 \mathrm{MeV}\right)$ or neutron $\left(M_{n}=939.57 \mathrm{MeV}\right)$ mass.

The isovector $\rho$ meson has a vector as well as a tensor coupling to the nucleon. The $\rho-\mathrm{N}$ interaction, which is involved in the Lagrangian density $\mathcal{L}_{N N \rho}$, is given by

$$
\mathcal{L}_{N N \rho}^{\text {int. }}=g_{\rho} \bar{\psi} \gamma^{\mu} \rho_{\mu} \tau_{z} \psi+i \frac{f_{\rho}}{2 M_{i}} \bar{\psi} \sigma^{\mu \nu} \partial_{\nu} \rho_{\mu} \tau_{z} \psi
$$

where $\psi$ and $\rho^{\mu}$ are, respectively, the fields of the nucleon and the neutral member of $\rho$ meson. Here $g_{\rho}$ and $f_{\rho}$ are the vector and tensor coupling constants, respectively.

To study the meson mixing amplitude, we first have to solve the nuclear ground state within RHA. In case of the $\rho$ and $\omega$ mesons, since the vector self-energy due to the nucleon loop vanishes in RHA [13, 14], the vacuum polarization does not change the energy density of the total system.

For the scalar mesons, it is necessary to treat the $\sigma-\delta$ mixing and the polarization corrections to the $\sigma$ - and $\delta$-meson propagators simultaneously. In Eq.(1), $\delta \mathcal{L}_{C T}$ involves counterterms such as $\sigma^{n} \delta^{m}(n, m=1 \sim 4)$, which remove the divergent pieces of the nucleon-loop contributions to the $\sigma-\delta$ mixing and the scalar-meson propagators. As in Ref. [13], the counterterms are determined so as to renormalize the polarization functions at $q_{\mu}^{2}=0$ and $M_{i}^{*}=M_{i}$. This prescription provides a finite vacuum fluctuation correction to the energy density of the total system. We finally find that in RHA the correction is given by a sum of $\Delta \mathcal{E}_{p}$ and $\Delta \mathcal{E}_{n}$, where

$$
\begin{aligned}
\Delta \mathcal{E}_{i} & =-\frac{1}{8 \pi^{2}}\left[M_{i}^{* 4} \ln \left(\frac{M_{i}^{*}}{M_{i}}\right)+M_{i}^{3}\left(M_{i}-M_{i}^{*}\right)-\frac{7}{2} M_{i}^{2}\left(M_{i}-M_{i}^{*}\right)^{2}\right. \\
& \left.+\frac{13}{3} M_{i}\left(M_{i}-M_{i}^{*}\right)^{3}-\frac{25}{12}\left(M_{i}-M_{i}^{*}\right)^{4}\right] .
\end{aligned}
$$

The total energy density in RHA is thus given by

$$
\mathcal{E}_{\text {tot }}=\mathcal{E}_{M F A}+\Delta \mathcal{E}_{p}+\Delta \mathcal{E}_{n}
$$

where $\mathcal{E}_{M F A}$ is the energy density in the mean-field approximation (MFA) [13]. In the present calculation, the $\sigma, \delta$ and $\rho$ masses are, respectively, taken to be $m_{\sigma}=550.0 \mathrm{MeV}, m_{\delta}=983.0$ $\mathrm{MeV}$ and $m_{\rho}=769.3 \mathrm{MeV}$. We determine $g_{\sigma}$ and $g_{\omega}$ (the $\omega-\mathrm{N}$ coupling constant) so as to 
fit the saturation condition for symmetric $(Z=N)$ nuclear matter: $\mathcal{E}_{\text {tot }} / \rho_{B}-M=-15.75$ $\mathrm{MeV}(M$, the average of the free proton and neutron masses) at the saturation density $\rho_{0}=0.17 \mathrm{fm}^{-3}$. Fixing the ratio of the tensor to vector coupling constants, $c_{\rho} \equiv f_{\rho} / g_{\rho}$, to be 5.0 [15], the vector coupling constant $g_{\rho}$ is chosen so as to reproduce the measured $\rho$ - $\omega$ mixing amplitude, $\left\langle\rho\left|H_{e m}\right| \omega\right\rangle=-4520 \mathrm{MeV}^{2}$, at the $\omega$ pole [2]. Finally, we determine $g_{\delta}$ so as to produce the empirical symmetry energy $a_{4}(=32.5 \mathrm{MeV})$ [16]. We then find that $g_{\sigma}=8.360, g_{\delta}=3.257, g_{\omega}=9.493$ and $g_{\rho}=3.455$. This yields the effective proton and neutron masses, $M_{p}^{*}=679.36 \mathrm{MeV}$ and $M_{n}^{*}=680.65 \mathrm{MeV}$, at $\rho_{0}$ and the nuclear incompressibility $K=460 \mathrm{MeV}$.

Now we are in a position to calculate meson mixing amplitudes in asymmetric $(Z \neq N)$ nuclear matter. There are four types of the CSB meson mixing: $\rho-\omega, \sigma-\delta, \sigma-\rho$ and $\delta-\omega$ mixing. In particular, the mixing of scalar and vector mesons (the latter two cases) is similar to the familiar $\sigma-\omega$ mixing in matter [14,17]. It is a purely density effect and forbidden in vacuum. Note that the $\rho-\omega$ mixing yields class III and IV forces while the others provide class III forces [1].

We first calculate the $\rho-\omega$ mixing. The polarization function due to the nucleon loop has a tensor character and it can be separated into the longitudinal (L) and transverse (T) components. They are identical each other in vacumm. The mixing amplitudes for the $\mathrm{L}$ and $\mathrm{T}$ modes are, respectively, given by the polarization functions as

$$
\left\langle\rho\left|H_{m i x}\right| \omega\right\rangle_{L}=\frac{q_{\mu}^{2}}{q_{s}^{2}} \Pi_{\rho \omega}^{00}\left(q_{0}, q_{s}\right) \quad \text { and } \quad\left\langle\rho\left|H_{m i x}\right| \omega\right\rangle_{T}=\frac{1}{2}\left[\Pi_{\rho \omega}^{11}\left(q_{0}, q_{s}\right)+\Pi_{\rho \omega}^{22}\left(q_{0}, q_{s}\right)\right]
$$

where we choose the direction of the meson momentum as $q^{\mu}=\left(q_{0}, 0,0, q_{s}\right)$ and

$$
\Pi_{\rho \omega}^{\mu \nu}\left(q_{0}, q_{s}\right)=-i g_{\omega} g_{\rho} \int \frac{d^{4} k}{(2 \pi)^{4}} \operatorname{Tr}\left[\gamma^{\mu} G(k+q) \tilde{\Gamma}_{\rho}^{\nu} \tau_{z} G(k)\right]
$$

Here $G$ is the nucleon propagator [13] and is a diagonal matrix in isospin space

$$
G(k)=\left(\begin{array}{cc}
G_{p}(k) & 0 \\
0 & G_{n}(k)
\end{array}\right) .
$$

As usual, the nucleon propagator can be devided into the Feynman (F) and densitydependent (D) parts [13].

In the $\rho$ - $\mathrm{N}$ tensor coupling in matter, it is natural to replace the free nucleon mass in Eq.(四) with the effective mass $M_{i}^{*}$ because it is originally derived by the Gordon decomposition of the nucleon field in matter1. Hence, in Eq.(8) we use the $\rho-\mathrm{N}$ coupling as

$$
\Gamma_{\rho}^{\mu}=\gamma^{\mu}-i \frac{c_{\rho}}{2 M_{i}^{*}} \sigma^{\mu \lambda} q_{\lambda} \quad \text { and } \quad \tilde{\Gamma}_{\rho}^{\mu}=\gamma^{\mu}+i \frac{c_{\rho}}{2 M_{i}^{*}} \sigma^{\mu \lambda} q_{\lambda}
$$

\footnotetext{
${ }^{1}$ This replacement of the nucleon mass is equivalent to adding terms such as $\sigma^{n} \bar{\psi} \sigma^{\mu \nu} \partial_{\nu} \rho_{\mu} \tau_{z} \psi$ and $\delta^{n} \bar{\psi} \sigma^{\mu \nu} \partial_{\nu} \rho_{\mu} \tau_{z} \psi(n=1,2, \cdots, \infty)$ to the original Lagrangian density. However, those terms do not modify the $\sigma$ and $\delta$ fields in RHA.
} 
Because of the isospin matrix $\tau_{z}$, the mixing amplitude is given by the difference between contributions from the proton and neutron loops. The polarization function which involves only the F part of $G$ becomes divergent. However, as in Ref. [7], by virtue of the replacement in Eq.(10) the difference between proton and neutron contributions becomes finite even in matter. The $\mathrm{F}$ part of the polarization function is then given by a sum of $\Pi_{v}^{F \mu \nu}$ and $\Pi_{t}^{F \mu \nu}$, where the former is the polarization function with the vector coupling at the $\rho$ - $\mathrm{N}$ vertex while the latter is that with the tensor coupling:

$$
\begin{aligned}
& \Pi_{v}^{F \mu \nu}\left(q_{\mu}^{2}\right)=\frac{\xi_{\mu \nu}}{2 \pi^{2}} g_{\omega} g_{\rho} q_{\mu}^{2} \int_{0}^{1} d x x(1-x) \ln \left[\frac{M_{p}^{* 2}-x(1-x) q_{\mu}^{2}}{M_{n}^{* 2}-x(1-x) q_{\mu}^{2}}\right], \\
& \Pi_{t}^{F \mu \nu}\left(q_{\mu}^{2}\right)=\frac{\xi_{\mu \nu}}{8 \pi^{2}} g_{\omega} f_{\rho} q_{\mu}^{2} \int_{0}^{1} d x \ln \left[\frac{M_{p}^{* 2}-x(1-x) q_{\mu}^{2}}{M_{n}^{* 2}-x(1-x) q_{\mu}^{2}}\right]
\end{aligned}
$$

with $\xi^{\mu \nu}=-g^{\mu \nu}+q^{\mu} q^{\nu} / q_{\mu}^{2}$. The polarization function involving at least one power of the $\mathrm{D}$ part of $G$ can be calculated analytically [14].

In the present calculation, we introduce a monopole-type form factor at each vertex in the spacelike region. Thus, we replace the coupling constant as

$$
g \rightarrow g\left(q_{\mu}^{2}\right)=g /\left(1-q_{\mu}^{2} / \Lambda^{2}\right),
$$

with the cutoff $\Lambda=1.5 \mathrm{GeV}$. Here we suppose that $\Lambda$ is common to all the vertices. (As pointed out in Ref. [10], properly speaking, it is necessary to calculate the CSB vertex from the same QHD theory that provided the propagator.)

In Fig. 1, we present the $\rho-\omega$ mixing amplitudes for the $\mathrm{L}$ and $\mathrm{T}$ modes in the spacelike region. We fix $q_{0}=0$ and vary $|\vec{q}|\left(=q_{s}\right)$. The nuclear density is chosen to be $\rho_{0}$. Here we introduce the proton fraction $f_{p}$ that is defined by $Z /(Z+N)$. In the figure, in addition to the case of symmetric nuclear matter $\left(f_{p}=0.5\right)$, the results for $f_{p}=0.49$ and 0.51 , which respectively correspond to the mirror nuclei of ${ }^{41} \mathrm{Ca}$ and ${ }^{41} \mathrm{Sc}$, are illustrated. The mixing amplitude in symmetric matter is very similar to that in vacuum. 2 However, in asymmetric matter the amplitude is much larger than that in symmetric case. The mixing amplitude for $f_{p}=0.51$ is opposite in sign relative to that for $f_{p}=0.49$ because the amplitude is given by the difference between proton and neutron contributions. It should be noticed that there is a sharp peak around $q_{s}=2 k_{F}^{i}\left(k_{F}^{i}\right.$, the Fermi momentum of proton or neutron) in the mixing amplitude and that the peak structure stems from the D part of the polarization function. As an example, in Fig. 22 we illustrate how such a peak is generated in the T mode with $f_{p}=0.51$. From the figure, we can see that the peak is produced by the cancellation of large amplitudes from the proton and neutron loops. In Fig. 3, as a function of $\rho_{B}$ and $f_{p}$, we show the ratio of the $\rho$ - $\omega$ mixing amplitude at the $\omega$ pole $\left(q_{0}=m_{\omega}\right.$ and $\left.q_{s}=0\right)$ in asymmetric matter to that in vacuum. Note that in the limit $q_{s} \rightarrow 0\left(q_{0} \neq 0\right)$ the L mode is identical to the $\mathrm{T}$ mode. The mixing amplitude at $\rho_{0}$ decreases gradually as $f_{p}$ increases. However, at high $\rho_{B}$ the amplitude changes drastically. In particular, at small $f_{p}$ and high $\rho_{B}$ the ratio becomes $30 \sim 40$. Such an extreme condition may correspond to the case of neutron stars [16,18.

\footnotetext{
${ }^{2}$ The present result in vacuum is consistent with the result calculated in Ref. [7].
} 
Next we consider the $\sigma-\delta$ mixing. The mixing amplitude (or the polarization function) is given by

$$
\left\langle\delta\left|H_{m i x}\right| \sigma\right\rangle=\Pi_{\sigma \delta}\left(q_{0}, q_{s}\right)=-i g_{\sigma} g_{\delta} \int \frac{d^{4} k}{(2 \pi)^{4}} \operatorname{Tr}\left[G(k+q) \tau_{z} G(k)\right] .
$$

The amplitude is again given by the difference between contributions from the proton and neutron loops. As mentioned above Eq.(5), the $\sigma-\delta$ mixing does not vanish in vacuum if $M_{p} \neq M_{n}$. The normalized $\mathrm{F}$ part of the polarization function is given by $\Pi_{\sigma \delta}^{F}\left(q_{\mu}^{2}\right)=$ $\Pi_{\sigma \delta p}^{F}\left(q_{\mu}^{2}\right)-\Pi_{\sigma \delta n}^{F}\left(q_{\mu}^{2}\right)$, where [19]

$$
\begin{aligned}
\Pi_{\sigma \delta i}^{F}\left(q_{\mu}^{2}\right) & =\frac{3 g_{\sigma} g_{\delta}}{4 \pi^{2}}\left(M_{i}^{2}+3 M_{i}^{* 2}-4 M_{i}^{*} M_{i}-\frac{1}{6} q_{\mu}^{2}\right. \\
& \left.-\int_{0}^{1} d x\left[M_{i}^{* 2}-x(1-x) q_{\mu}^{2}\right] \ln \left[\frac{M_{i}^{* 2}-x(1-x) q_{\mu}^{2}}{M_{i}^{2}}\right]\right) .
\end{aligned}
$$

The $\mathrm{D}$ part of the polarization function can be calculated explicitly. It is similar to the $\mathrm{D}$ part of the nucleon-loop contribution to the $\sigma$-meson propagator, except for the isospin matrix $\tau_{z}$ [14.

For the scalar-vector $(\sigma-\rho$ or $\delta$ - $\omega)$ meson mixing, the mixing amplitude (or the polarization function) is given by

$$
\left\langle v\left|H_{m i x}\right| s\right\rangle=\Pi_{s v}\left(q_{0}, q_{s}\right)=i g_{s} g_{v} \delta_{0 \mu} \int \frac{d^{4} k}{(2 \pi)^{4}} \operatorname{Tr}\left[G(k+q) \tilde{\Gamma}_{v}^{\mu} \tau_{z} G(k)\right]
$$

where $s$ and $v$ stand for the scalar and vector mesons, respectively (if $v=\omega, \tilde{\Gamma}_{\omega}^{\mu}=\gamma^{\mu}$ ). As in the $\sigma-\omega$ mixing [14, [17], this amplitude vanishes identically in vacuum and hence is a purely density effect. The polarization function can be calculated analytically 14.

In Fig. $₫$, we present the results of the $\sigma-\delta, \sigma-\rho$ and $\delta-\omega$ mixing in asymmetric nuclear matter. The behavior of the $\sigma-\delta$ mixing is very similar to that of the $\delta-\omega$ mixing. However, the $\sigma-\delta$ mixing amplitude is opposite in sign compared with the $\delta$ - $\omega$ mixing. By contrast, in the $\sigma-\rho$ mixing the sign of the amplitude changes at $q_{s} \simeq 0.63 M$. This is due to the $\rho$-N tensor coupling that dominates for large $q_{s}$. In the top panel of the figure, the $\sigma-\delta$ mixing amplitude in vacuum is also shown by the dotted curve. It may also contribute to the NN scattering lengths in free space [1].

In Fig. 5, we illustrate the meson mixing amplitues in $\mathrm{Pb}\left(\rho_{B}=0.15 \mathrm{fm}^{-3}\right.$ and $\left.f_{p}=0.4\right)$ and neutron stars. We suppose that in neutron stars the nuclear density is about $3 \rho_{0}$ and $f_{p} \sim 0.15$ 16, 18]. From the figure we can see that in $\mathrm{Pb}$ the mixing amplitudes are about $5 \sim 10$ times as large as those in matter with $f_{p}=0.49$ (see Figs. 1 and 1). Furthermore, in neutron stars the $\rho-\omega$ mixing amplitude in the $\mathrm{L}$ mode and the $\sigma$ - $\rho$ mixing amplitude are about 100 times larger than those for $f_{p}=0.49$. This fact is consistent with the result shown in Fig. 3 .

In summary, using QHD we have studied the $\rho-\omega, \sigma-\delta, \sigma-\rho$ and $\delta$ - $\omega$ mixing amplitudes in isospin asymmetric nuclear matter. We have shown that the mixing amplitude is quite sensitive to the proton fraction and nuclear density. In particular, in neutron stars and/or neutron rich nuclei (like $\mathrm{Pb}$ ) the CSB meson mixing amplitudes become considerably large compared with those in typical mirror nuclei. Even in light nuclei, if $f_{p}$ is far from 0.5 
(like halo nuclei [20]), the CSB meson mixing would play an important role in the nuclear structure. In the present calculation, we have not calculated the CSB potential generated by the meson mixing because, in addition to the mixing amplitude, it is necessary to know the CSB meson-N vertex to draw any definite conclusions on the potential [10]. Such a complete study requires more elaborate calculations and it is beyond the scope of this Letter. It would clearly be very interesting and important to calculate it in the future.

We would like to thank A. Suzuki, A.W. Thomas and A.G. Williams for valuable discussions. 


\section{REFERENCES}

[1] E.M. Henley, G.A. Miller, in: Mesons in nuclei, edited by M. Rho and D.H. Wilkinson (North-Holland, Amsterdam, 1979), p.405;

G.A, Miller, B.M.K. Nefkens, I. Šlaus, Phys. Rep. 194 (1990) 1;

G.A. Miller, W.T.H. Van Oers, in: Symmetries and fundamental interactions in nuclei, edited by W.C. Haxton and E.M. Henley (World Scientific, Singapore, 1995), p.127.

[2] L.M. Barkov et al., Nucl. Phys. B256 (1985) 365;

S.A. Coon, R.C. Barret, Phys. Rev. C36 (1987) 2189.

[3] S. Shlomo, Rep. Prog. Phys. 41 (1978) 957;

P.G. Blunden, M.J. Iqbal, Phys. Lett. B198 (1987) 14;

K. Saito, A.W. Thomas, Phys. Lett. B335 (1994) 17;

M. Kimura, A. Suzuki, H. Tezuka, Phys. Lett. B367 (1996) 5;

K. Tsushima, K. Saito, A.W. Thomas, Phys. Lett. B465 (1999) 36.

[4] R. Abegg, Phys. Rev. Lett. 56 (1986) 2571; Phys. Rev. D39 (1989) 2464.

[5] L.D. Knutson, Phys. Rev. Lett. 66 (1991) 1410;

S.E. Vigdor, Phys. Rev. C46 (1992) 410.

[6] T. Goldman, J.A. Henderson, A.W. Thomas, Few Body Syst. 12 (1992) 193;

G. Krein, A.W. Thomas, A.G. Williams, Phys. Lett. B317 (1993) 293

H.B. O'Connell, B.C. Pearce, A.W. Thomas, A.G. Williams, Phys. Lett. B336 (1994) 1.

[7] J. Piekarewicz, A.G. Williams, Phys. Rev. C47 (1993) R2462.

[8] T. Hatsuda, E.M. Henley, Th. Meissner, G. Krein, Phys. Rev. C49 (1994) 452.

[9] S.A. Coon, B.H.J. McKeller, A.A. Rawlinson, in: Intersections between particle and nuclear physics, edited by T.W. Donelly, AIP conf. proc. no.412 (AIP, N.Y., 1997), p.368.

[10] T.D. Cohen, G.A. Miller, Phys. Rev. C52 (1995) 3428.

See also, S. Gardner, C.J. Horowitz, J. Piekarewicz, Phys. Rev. C53 (1996) 1143.

[11] H.B. O'Connell, B.C. Pearce, A.W. Thomas, A.G. Williams, Prog. Part. Nucl. Phys. 39 (1997) 201.

[12] For the timelike region, see, for example, A.K. Dutt-Mazumder, Nucl. Phys. A611 (1996) 442.

[13] B.D. Serot, J.D. Walecka, Adv. Nucl. Phys. 16 (1986) 1.

[14] K. Saito, K. Tsushima, A.W. Thomas, A.G. Williams, Phys. Lett. B433 (1998) 243;

K. Lim, C.J. Horowitz, Nucl. Phys. A501 (1989) 729.

[15] R. Machleidt, in Proceedings of the relativistic dynamics and quark-nuclear physics, edited by M.B. Johnson and A. Picklesimer (Wiley, New York, 1986);

J.J. Sakurai, in Currents and mesons (Univ. of Chicago Press, Chicago, 1969).

[16] N.K. Glendenning, F. Weber, S.A. Moszkowski, Phys. Rev. C45 (1992) 844.

[17] S.A. Chin, Ann. of Phys. (N.Y.) 108 (1977) 301.

[18] F. Weber, astro-ph/0207053;

C.J. Horowitz, J. Piekarewicz, nucl-th/0207067.

[19] R.J. Furnstahl, C.J. Horowitz, Nucl. Phys. A485 (1988) 632.

[20] I. Tanihata, Nucl. Phys. A654 (1999) 235c. 


\section{FIGURES}
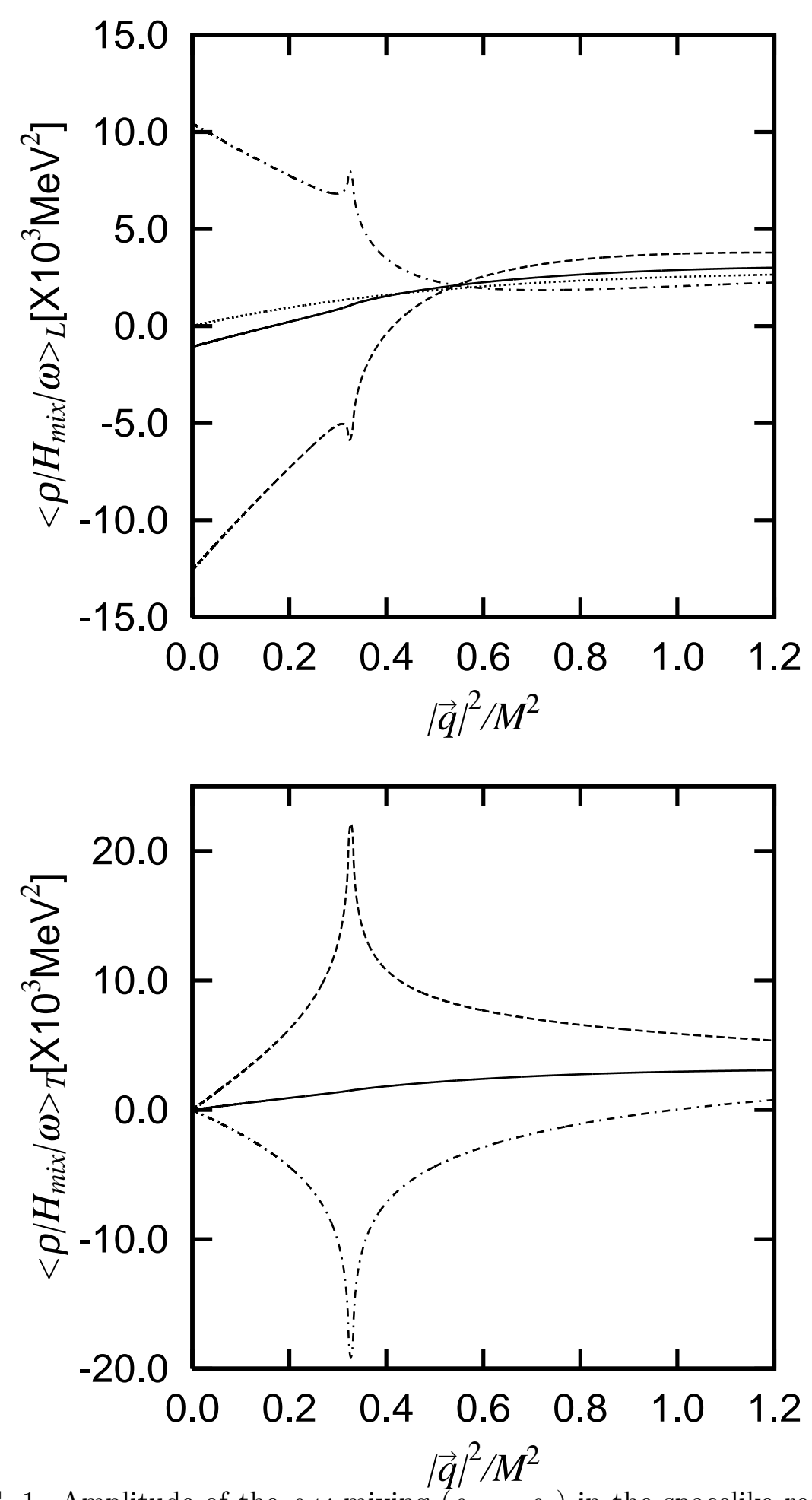

FIG. 1. Amplitude of the $\rho-\omega$ mixing $\left(\rho_{B}=\rho_{0}\right)$ in the spacelike region. We take $q_{0}=0$ and vary $|\vec{q}|\left(=q_{s}\right)$. The top panel is for the $\mathrm{L}$ mode while the bottom one is for the $\mathrm{T}$ mode. The solid (dashed) [dot-dashed] curve represents the result with $f_{p}=0.5(0.49)[0.51]$ while the result in vacuum is shown by the dotted curve in the top panel. 


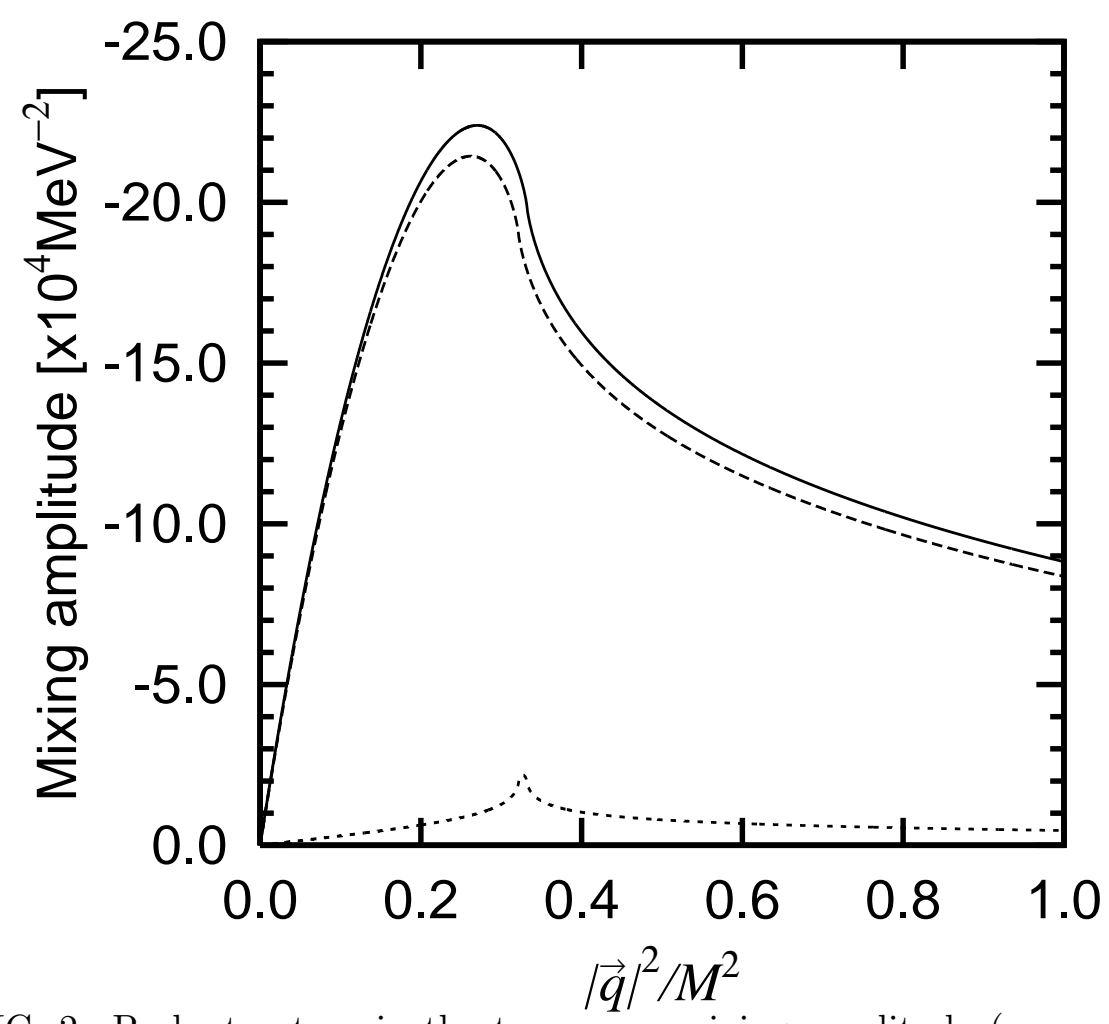

FIG. 2. Peak structure in the transverse mixing amplitude $\left(\rho_{B}=\rho_{0}\right.$ and $\left.f_{p}=0.51\right)$. The solid (dashed) curve represents the mixing amplitude due to the proton (neutron) loop while the difference between proton and neutron contributions are shown by the short-dashed curve. 


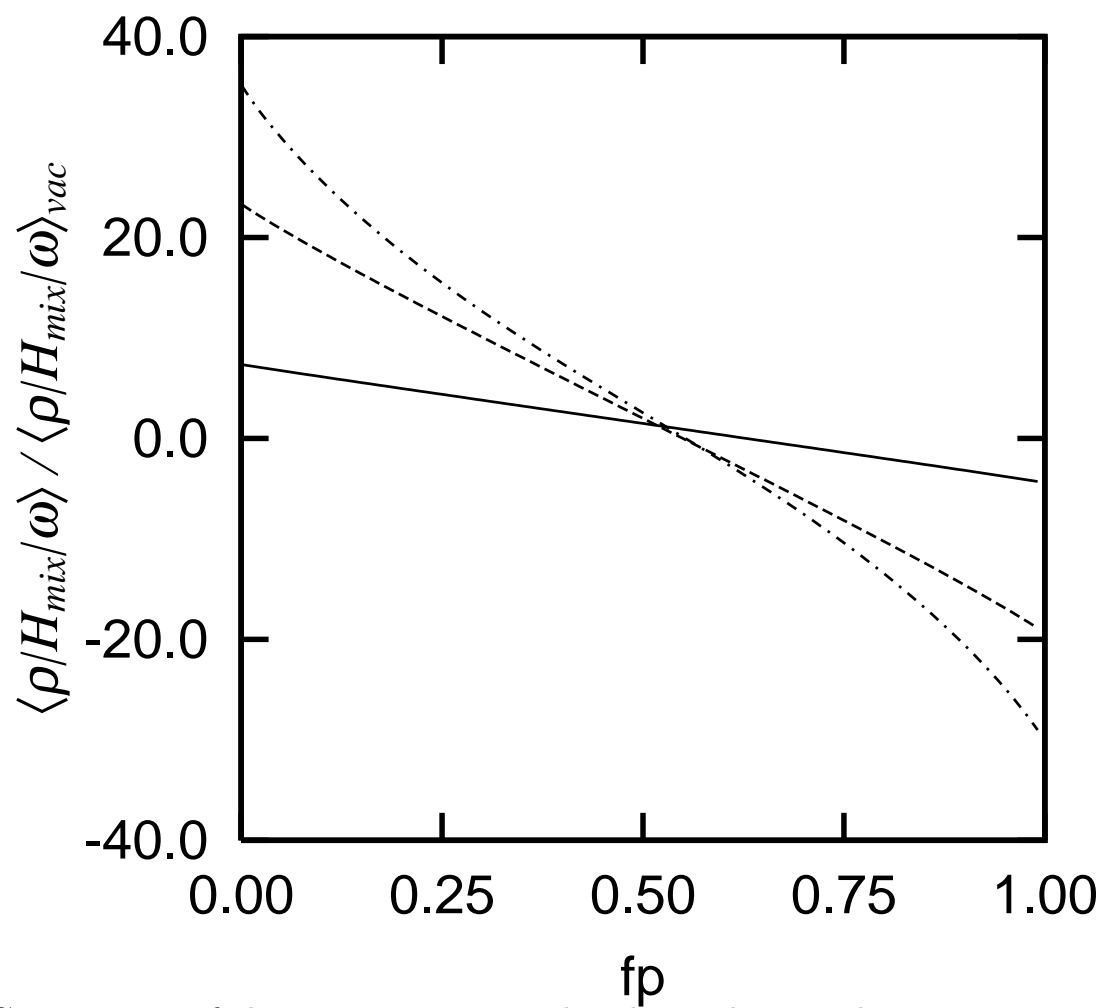

FIG. 3. Ratio of the $\rho$ - $\omega$ mixing amplitude at the $\omega$ pole in asymmetric nuclear matter to that in vacuum. The solid (dashed) [dot-dashed] curve is for $\rho_{0}\left(2 \rho_{0}\right)\left[3 \rho_{0}\right]$. 

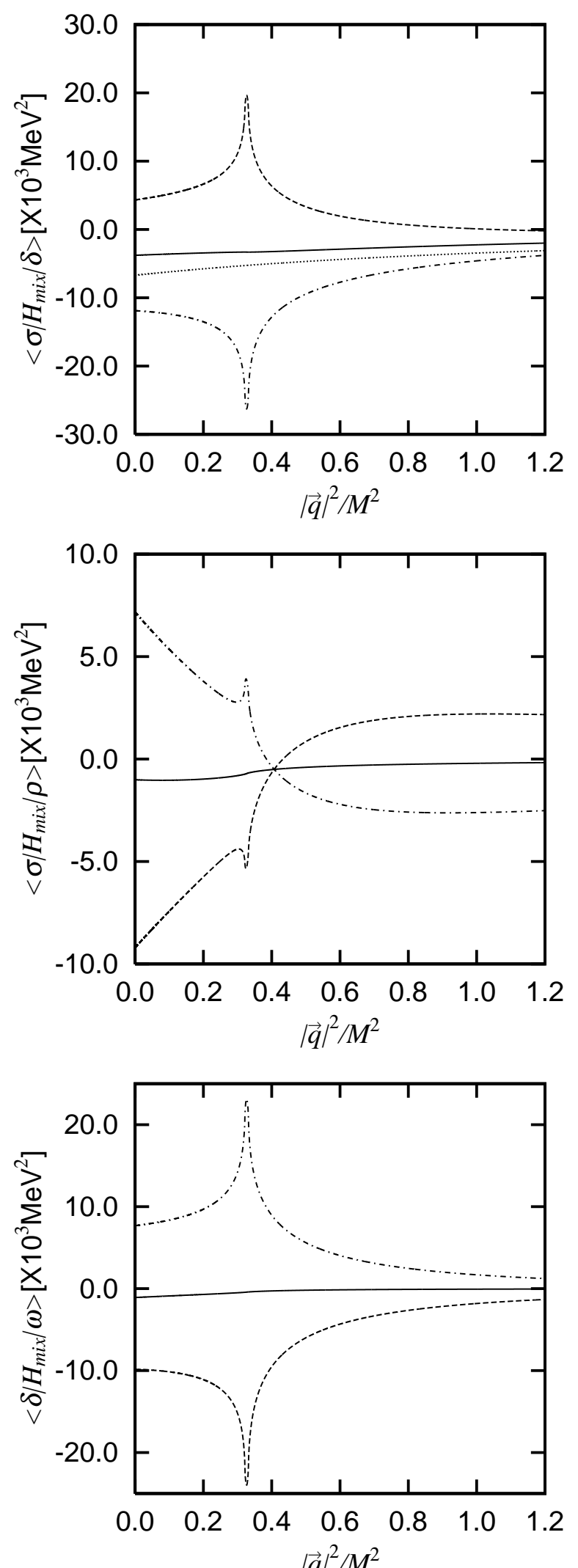

FIG. 4. Same as Fig. 1 but for the $\sigma-\delta$ (top panel), $\sigma-\rho$ (middle panel) and $\delta$ - $\omega$ (bottom panel) mixing amplitudes $\left(\rho_{B}=\rho_{0}\right)$. 

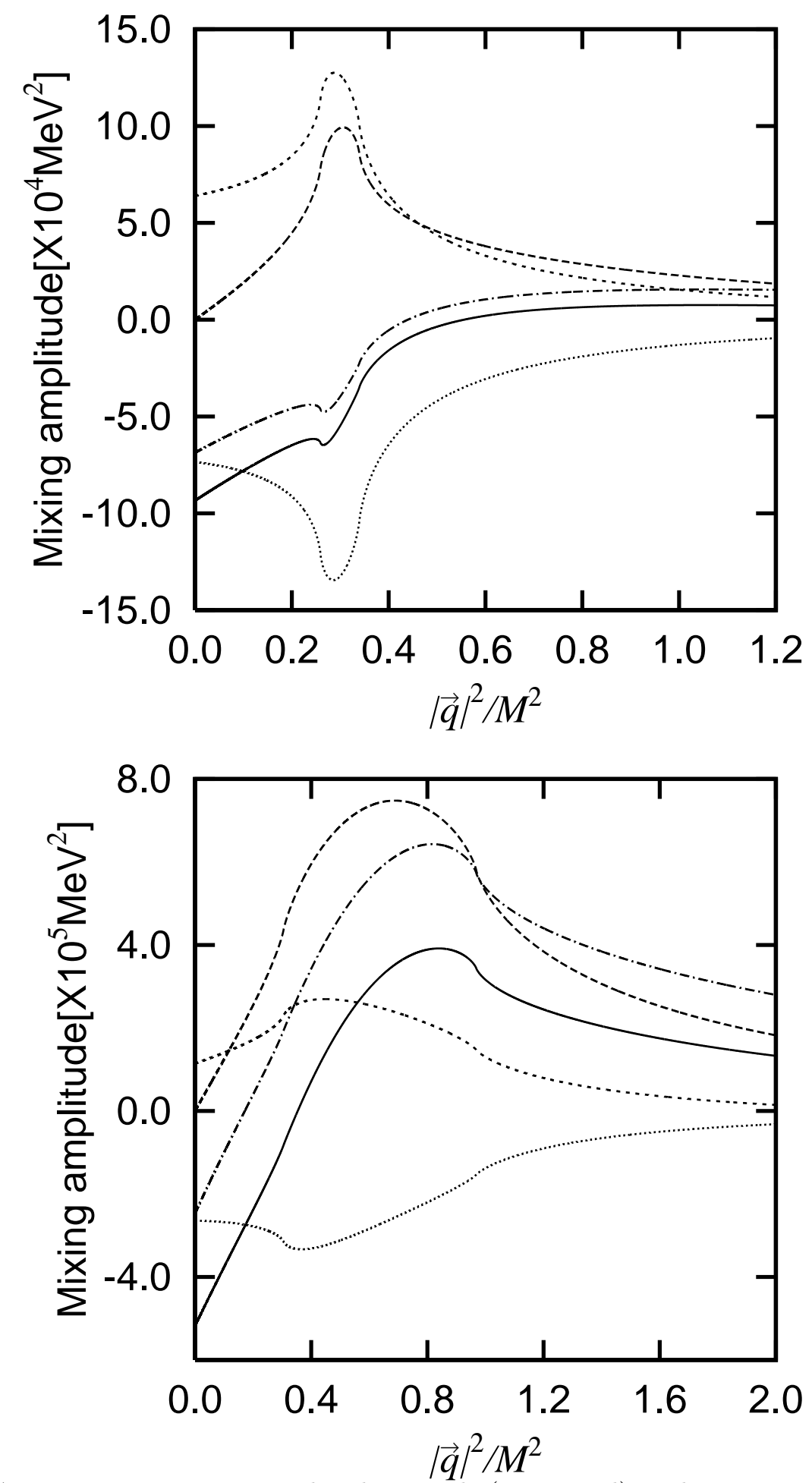

FIG. 5. Meson mixing amplitudes in $\mathrm{Pb}$ (top panel) and neutron stars (bottom panel). We take $\rho_{B}=0.15 \mathrm{fm}^{-3}$ and $f_{p}=0.4$ for $\mathrm{Pb}$ while $\rho_{B}=3 \rho_{0}$ and $f_{p}=0.15$ for neutron stars. The $\mathrm{L}$ (T) mode of the $\rho-\omega$ mixing is shown by the solid (long-dashed) curve. The short-dashed (dotted) [dot-dashed] curve is for the $\sigma-\delta(\delta-\omega)[\sigma-\rho]$ mixing. 